\title{
12例努南综合征患儿的临床特征及重组人生长 激素疗效观察
}

上官华坤,徐源涁,陈瑞敏

福建医科大学附属福州儿童医院内分泌遗传代谢科,福建 福州 350001

\begin{abstract}
[摘 要] 目的: 探讨努南综合征患儿的临床表现和遗传学特点, 总结重组人生长 激素的治疗效果。方法: 收集 2015 年 9 月至 2021 年 4 月就诊于福建医科大学附属福 州儿童医院经基因检测确诊的 12 例努南综合征患儿的资料。以身高低于 2 个标准 差或第3百分位为身材矮小的评判标准, 对 7 例患儿进行重组人生长激素 $(\mathrm{rhGH})$ 治 疗, 每 3 个月随访一次。结果: 12 例患儿均表现为特殊面容, 身材矮小 11 例, 先天性 心脏病 5 例, 面部雀斑 4 例, 咖啡斑 3 例, 智力障碍 3 例, 隐粹 3 例, 喂养困难 2 例, 骨骼 畸形包括脊柱侧弯 2 例、鸡胸 2 例、漏斗胸 1 例, 肋骨发育不良合并短指 1 例, 远视 1 例, 近视 1 例, 青春发育期提前 1 例。基因检测结果显示, 10 例患儿携带PTPN11基 因突变, 1 例患儿携带 $R I T 1$ 基因突变, 1 例患儿携带 $R A F 1$ 基因突变, 所有突变均分类 为致病性或可能致病性。7 例患儿 rhGH 平均治疗时间为 $(1.5 \pm 0.8)$ 年。与治疗前 比较, $\mathrm{rhGH}$ 治疗后患儿生长速率增加 [治疗前 $(2.8 \pm 1.0) \mathrm{cm} /$ 年, 治疗后 $(8.3 \pm$ $1.6) \mathrm{cm} /$ 年, $P<0.01]$, 治疗 $3 \sim 6$ 个月时生长速率最大, 此后随着治疗时间的延长生 长速率逐渐减小。7例患儿治疗期间胰岛素样生长因子 1 水平较治疗前均增加, 但 仍维持在正常范围。结论: 努南综合征临床表型谱广, 涉及全身多个系统, 基因检 测可明确诊断。身材矮小的努南综合征患儿 rhGH治疗可提高生长速率、改善身 高, 且治疗期间未见明显不良反应。
\end{abstract}

[关键词] 努南综合征; 基因突变; 身材矮小症; 青春发育期提前; 治疗结果; 随访研究

[ 中图分类号 ］R596［文献标志码 ］ A

\section{Clinical features, genetic detection and therapeutic response to rhGH of children with Noonan syndrome: an analysis of 12 cases}

SHANGGUAN Huakun, XU Yuanbin, CHEN Ruimin (Department of Endocrine Genetics and Metabolism, Fuzhou Children' Hospital of Fujian Medical University, Fuzhou

收稿日期:2021-05-11 接受日期:2021-07-26

基金项目:福州市临床重点专科建设项目(201610191); 福州市临床医学中心建设项目(2018080310)

第一作者: 上官华坤, 住院医师, 主要从事小儿内分泌遗传学研究;E-mail: 1249652614@qq.com; https ://orcid.org/00000002-4420-2232

通信作者: 陈瑞敏, 主任医师, 主要从事小儿内分泌遗传学研究;E-mail : chenrm321@sina.com; https://orcid.org/0000-00016586-0544 
350001, China)

Corresponding author: CHEN Ruimin, E-mail: chenrm321@sina.com, https:/orcid.org/ 0000-0001-6586-0544

\begin{abstract}
[ Abstract ] Objective: To analyze the clinical manifestations, genetic features and therapeutic efficacy of patients with Noonan syndrome (NS). Methods: The clinical data of 12 NS children treated in Fuzhou Children' Hospital of Fujian Medical University from September 2015 to April 2021 were analyzed. Among them, 7 patients with height lower than two standard deviations of the mean (or below the third percentile) were treated with recombinant human growth hormone $(\mathrm{rhGH})$, and were followed up every 3 months. Results: The clinical characteristics were as following: facial anomalies $(n=12)$, short stature $(n=11)$, congenital heart diseases $(n=5)$, facial freckles $(n=4)$, coffee spots on the skin $(n=3)$, intelligence disability $(n=3)$, cryptorchidism $(n=3)$, feeding difficulties $(n=2)$, scoliosis $(n=2)$, pectus carinatum $(n=2)$, pectus excavatum $(n=1)$, rib dysplasia companied with short finger $(n=1)$, hyperopia $(n=1)$, myopia $(n=1)$ and early puberty $(n=1)$. The PTPN11 mutation was detected in 10 cases, RIT1 mutation was detected in 1 case, and RAF1 mutation was detected in 1 case. In 7 patients treated with rhGH, the mean height velocity increased from $(2.8 \pm 1.0) \mathrm{cm} /$ year before treatment to $(8.3 \pm 1.6) \mathrm{cm} /$ year after treatment for $(1.5 \pm 0.8)$ years $(P<0.01)$; the height velocity was the fastest during 3 to 6 months of treatment, and then gradually went slower. The serum levels of insulin-like growth factor 1 (IGF-1) remained within the normal range. Conclusions: The clinical manifestations of NS are diverse, and the disease can be diagnosed through genetic testing. For NS patients with short stature, rhGH treatment can increase the height velocity and no obvious adverse reactions were found.
\end{abstract}

[ Key words ] Noonan syndrome; Gene mutation; Short stature; Early puberty; Treatment outcome; Follow-up studies

[J Zhejiang Univ (Med Sci), 2021, 50(4): 500-505.]

[缩略语］肾素一血管紧张素系统(renin-angiotensin system, Ras); 丝裂原激活蛋白激 酶(mitogen activation protein kinase,MAPK); 重组人生长激素(recombinant human growth hormone,rhGH); 胰岛素样生长因子(insulin-like growth factor,IGF); 磁共振成 像 (magnetic resonance imaging, MRI); 促性腺激素释放激素 (gonadotropin releasing hormone, GnRH)

努南综合征是一种罕见的先天性畸形综合 征, 因 Ras/MAPK信号通路相关基因突变所致, 目 前研究显示有 16 个基因的突变与该病的发病相关, 以PTPN11(50\%)、SOS1(20\%)和RAF1(5\% 15\%)较 为常见 ${ }^{[1]}$ 。努南综合征的临床表现涉及心脏、骨 骼、泌尿、消化及内分泌等多个系统异常, 以特殊面 容、身材矮小、先天性心脏病、男性隐睪和骨骼畸形 最为多见, 癫㾁、视力异常和皮肤色素沉着亦较为
常见,部分患儿还可能出现青春发育延迟 ${ }^{[1-2]}$ 。 研究显示,身材矮小的努南综合征患儿成年 终身高低于平均身高 2 个标准差或第 3 个百分位 数 ${ }^{[3-5]}$ 。国外的一些观察性证据表明, rhGH可增 加努南综合征矮小患儿在治疗期间的生长速率和 缩小身高标准差 ${ }^{[6-7]}$ 。目前, 美国食品药品监督管 理局和中华医学会儿科学分会内分泌遗传代谢学 组推荐 rhGH用于治疗合并身材矮小的努南综合 
征患儿 ${ }^{[8]}$, 然而关于 $\mathrm{rhGH}$ 治疗的有效性和安全性 数据较少,国内仅刘之慧等 ${ }^{[9]}$ 报道分析 5 例患儿接 受 $\mathrm{rhGH}$ 治疗 1 年的效果。本文回顾性分析了我们 近年收治的 12 例努南综合征患儿的临床资料以及 接受 $r h G H$ 治疗患儿的疗效, 为临床医师更好地了 解努南综合征提供参考。

\section{1 对象与方法}

\section{1 对 象}

收集 2015 年 9 月至 2021 年 4 月就诊于福建医 科大学附属福州儿童医院内分泌遗传代谢门诊经 基因检测确诊的 12 例努南综合征患儿的资料。其 中, 男性 8 例, 女性 4 例, 平均年龄 $(4.7 \pm 2.4)$ 岁。 所有患儿的诊断均符合临床实践指南 ${ }^{[1]}$, 即具有 努南综合征表型的患儿检测到致病或可能致病基 因突变。研究获得所有受试患儿家属的知情同 意, 并通过福建医科大学附属福州儿童医院伦理 委员会批准 $[2019$ (伦审)第 21 号]。

\section{2 实验室及影像学检查}

抽取患儿空腹静脉血 $3 \mathrm{~mL}$, 高速离心后取血. 清, 采用西门子全自动化学发光免疫分析仪 (Immulite 2000)检测生长激素、IGF-1、IGF结合蛋 白3、甲状腺和肾上腺及性腺轴激素、空腹胰岛素、 C 肽、糖化血红蛋白。采用德国 Siemens 公司生产 的直接数字化拍片机和德国 Agfa 计算机X线摄影 系统拍摄骨龄。行垂体和/或头颖 MRI、心脏、上腹 部及妇科彩色多普勒超声检查。

\section{3 基因检测及其致病性分析}

采集患儿及父母静脉血 $2 \mathrm{~mL}$, 加人乙二胺四 乙酸抗凝, $4{ }^{\circ} \mathrm{C}$ 保存。委托上海交通大学医学院附 属上海儿童医学中心、贝瑞基因公司及康旭医学 检验所进行基因检测。使用Blood Genomic DNA Mini Kit 血液基因组 DNA 柱式小量提取试剂盒 (CW2087M, 康为世纪) 从外周血抽提所有标本基 因组DNA。采用SureSelect Human All Exon V6试 剂盒(美国Aligent公司)建库, Novaseq 6000 系统 (美国Illumina公司)进行高通量测序。检索 ClinVar数据库 (https://www. ncbi. nlm. nih. gov/ clinvar/), 结合美国医学遗传学与基因组学学会联 合分子病理协会提出的 “序列变异解读标准和指 南” 分析致病性 ${ }^{[10]}$ 。

\section{1. $4 \mathrm{rhGH}$ 治疗及随访}

身高低于 2 个标准差或第 3 百分位的患儿接
受 $r h G H$ 治疗 ${ }^{[8]}$ 。最终纳人 7 例患儿进行 $r h G H$ 治 疗, 以 $0.10 \sim 0.15 \mathrm{IU} \cdot \mathrm{kg}^{-1} \cdot \mathrm{d}^{-1}$ (皮下注射)为起 始剂量, 之后每隔 3 个月进行一次随访, 随访内容 包括治疗后的不良反应、体格检查(身高和体 重)、实验室检查 (血尿常规、肝肾功能、甲状腺功 能、 IGF-1)。根据治疗反应及 $\mathrm{IGF}-1$ 水平调整 剂量。

\section{5 统计学方法}

使用GraphPad Prism 8.0软件进行统计学分 析。符合正态分布的计量资料以均数 \pm 标准差 $(\bar{x} \pm s)$ 表示, 采用 $t$ 检验。 $P<0.05$ 为差异有统计学 意义。

\section{2 结 果}

\subsection{2例努南综合征患儿临床特征}

患儿均有特殊面容, 以眼距宽 $(10 / 12)$ 、上睑 下垂 $(9 / 12)$ 、内䀝赘皮 $(6 / 12)$ 、前额宽 $(5 / 12)$ 及耳 位低 $(5 / 12)$ 常见,其他包括三角脸 $(3 / 12)$ 、睑裂下 斜 $(3 / 12)$ 、鼻梁低 $(3 / 12)$ 、小下领 $(3 / 12)$ 、高腭弓 $(2 / 12)$ 、颈蹼 $(2 / 12)$ 、耳位后旋 $(1 / 12)$ 、颈短 $(1 /$ $12)$ 和颈前倾 $(1 / 12)$; 身材矮小 11 例、偏矮 1 例, 先 天性心脏病 5 例, 面部雀斑 4 例、咖啡斑 3 例, 智力 障碍 3 例, 隐睪 3 例、生长激素缺乏 2 例, 喂养困难 2 例, 脊柱侧弯 2 例、鸡胸 2 例、漏斗胸 1 例、肋骨发 育畸形合并短指 1 例, 近视 1 例、远视 1 例, 青春发 育期提前 1 例。所有患儿垂体 MRI 检查均未见异 常。结果提示, 努南综合征患儿临床表现多样,除 特殊面容外常伴多系统发育异常。

\subsection{2 例努南综合征患儿基因突变情况}

12 例患儿基因突变情况见表 1 。10例患儿存 在PTPN11 基因突变、 1 例 RIT 1 基因突变、 1 例 $R A F 1$ 基因突变,所有突变在 ClinVar 数据库中均有报 道,且分类均为致病性或可能致病性。除 2 例患儿 的父母未接受检测 ,其余 10 例患儿携带的基因突 变源于母亲 1 例,携带的基因突变源于父亲 2 例, 另7例患儿的突变均为新发突变。结果提示, 本文 资料努南综合征基因突变以 PTPN11常见,且以新 发突变为主。

\subsection{7 例努南综合征患儿rhGH治疗效果}

7例患儿接受 $\mathrm{rhGH}$ 治疗。开始治疗的平均年 龄为 $(6.3 \pm 2.2)$ 岁, 平均出生身长为 $(50.1 \pm$ $1.4) \mathrm{cm}$, 平均出生体重为 $(3.0 \pm 0.4) \mathrm{kg}$ 。2例患儿 合并生长激素缺乏。7例患儿 rhGH 治疗的平均剂 
表 1 12例努南综合征患儿基因突变情况

Table 1 Gene mutations in 12 patients with Noonan syndrome

\begin{tabular}{clllll}
\hline 例 序 & 基因型 & 核苷酸位点 & 氨基酸位点 & 来 源 & 致病评级 \\
\hline 1 & $P T P N 11$ & c. $1529 \mathrm{~A}>\mathrm{G}$ & p. Q510R & 母亲 & 致病 \\
2 & $P T P N 11$ & c. $844 \mathrm{~A}>\mathrm{G}$ & p. $282 \mathrm{~V}$ & 父亲 & 致病 \\
3 & $P T P N 11$ & c. $184 \mathrm{~T}>\mathrm{G}$ & p. $\mathrm{Y} 62 \mathrm{D}$ & 新发 & 致病 \\
4 & $P T P N 11$ & c. $794 \mathrm{G}>\mathrm{A}$ & p. $\mathrm{R} 265 \mathrm{Q}$ & 新发 & 致病 \\
5 & $P T P N 11$ & c. $1508 \mathrm{G}>\mathrm{A}$ & p. $\mathrm{G} 503 \mathrm{E}$ & 新发 & 致病 \\
6 & $P T P N 11$ & c. $188 \mathrm{~A}>\mathrm{G}$ & p. $\mathrm{Y} 63 \mathrm{C}$ & 新发 & 致病 \\
7 & $P T P N 11$ & c. $188 \mathrm{~A}>\mathrm{G}$ & p. $\mathrm{Y} 63 \mathrm{C}$ & 新发 & 致病 \\
8 & RIT1 & c. $270 \mathrm{G}>\mathrm{A}$ & p. M90I & 未验证 & 可能致病 \\
9 & $P T P N 11$ & c. $922 \mathrm{~A}>\mathrm{G}$ & p. $\mathrm{N} 308 \mathrm{D}$ & 父亲 & 致病 \\
10 & $P T P N 11$ & c. $836 \mathrm{~A}>\mathrm{G}$ & p. Y279C & 新发 & 致病 \\
11 & $P T P N 11$ & c. $922 \mathrm{~A}>\mathrm{G}$ & p. $\mathrm{N} 308 \mathrm{D}$ & 未验证 & 可能致病 \\
12 & $R A F 1$ & c. $770 \mathrm{C}>\mathrm{T}$ & p. S257L & 新发 & 致病 \\
\hline
\end{tabular}

量为 $(0.130 \pm 0.014) \mathrm{IU} \cdot \mathrm{kg}^{-1} \cdot \mathrm{d}^{-1}$, 治疗时间为 6 个月 3 年, 平均 $(1.5 \pm 0.8)$ 年。与治疗前比较, $\mathrm{rhGH}$ 治疗后患儿生长速率增加 [治疗前 $(2.8 \pm$ $1.0) \mathrm{cm} /$ 年, 治疗后 $(8.3 \pm 1.6) \mathrm{cm} /$ 年, $P<0.01]$, 治疗 3 6个月时生长速率最大, 此后随着治疗时间 的延长生长速率逐渐减小。相比之下, 5 例未接受 $\mathrm{rhGH}$ 治疗的患儿就诊前平均生长速率为 $3 \sim 4 \mathrm{~cm} /$ 年,其中 2 例接受随访, 1 例 2 岁 6 个月初诊时身高 为 $83.5 \mathrm{~cm}(-2.11$ 个标准差), 3 岁复诊时身高为 $88.3 \mathrm{~cm}$ (-2.11个标准差); 另外 1 例 5 岁 10 个月初 诊时身高为 $101.3 \mathrm{~cm}(-3.36$ 个标准差), 7 岁 3 个 月复诊时身高为 $107.0 \mathrm{~cm}$ ( -3.32 个标准差), 均保 持原来生长速率。7例接受 $\mathrm{rhGH}$ 治疗的患儿治疗 期间 IGF-1 较治疗前均增加, 但仍维持在正常范 围。结果提示, rhGH 治疗能够提高努南综合征矮 小患儿在治疗期间的生长速率。

\subsection{7 例努南综合征患儿rhGH 治疗后随访结果}

7例接受 rhGH 治疗的患儿血糖、甲状腺功能 保持正常, 无颖内压增高相关的头痛、视力下降症 状, 无脊柱侧弯、关节增大及水电解质失衡等不良 事件。例 1 治疗半年后停药 4 年, 于 9.5 岁复诊, 体 检睪丸 $10 \mathrm{~mL}$,追问病史,家长未注意性征出现变 化时间, 予完善 $\mathrm{GnRH}$ 激发试验, 结果显示, 促黄体 生成素峰值为 $26.5 \mathrm{IU} / \mathrm{L}$, 促卵泡激素峰值为 $8.40 \mathrm{IU} / \mathrm{L}$, 骨龄为 10.8 岁, 考虑为努南综合征合 并青春发育期提前。其他 6 例患儿性征均维持在 青春期前状态。结果提示, 努南综合征患儿接受 $\mathrm{rhGH}$ 治疗期间未见明显不良反应, 在随访过程中
需要注意性征变化。

\section{3 讨 论}

努南综合征的发病机制与细胞Ras/MAPK通 路相关基因突变导致该通路信号上调有关。迄今 已报道 16 个 Ras/MAPK通路相关基因的突变与努 南综合征有关, 其中以PTPN11最常见 ${ }^{[1]}$ 。努南综 合征的临床表现多样,除特殊面容外常伴多系统 发育异常, 如身材矮小、先天性心脏病、男性隐睪、 骨骼畸形等。本文资料中的 12 例患儿符合努南综 合征的临床表型,其中 10 例携带PTPN11基因突 变, 另外 2 例分别携带 $R I T 1$ 和 $R A F 1$ 基因突变。

青春发育延迟是努南综合征患儿的典型表 现 $^{[2]}$, 既往文献报道中未见携带PTPN11 基因 c. $1529 \mathrm{~A}>\mathrm{G}$ 突变的努南综合征患儿出现性早熟或 青春发育期提前 ${ }^{[11-12]}$ 。本文资料中 1 例患儿出现 青春发育期提前,患儿垂体MRI正常,可排除继发 于中枢性肿瘤等器质性疾病, 而 Ras/MAPK通路 是参与 $\mathrm{GnRH}$ 受体信号传导级联调控的途径之一, 该途径的遗传异常可能导致青春发育延迟或中枢 性性早熟, 近年来有较多的证据显示中枢性性早 熟或青春发育期提前与 Ras/MAPK 通路异常相关 疾病联系密切 ${ }^{[13-15]}$ 。本文资料中的这 1 例患儿和 已有报道 9 例中的 8 例患儿 ${ }^{[14-15]}(88.9 \%)$ 均为男 性, 且所有患儿的垂体 MRI 均未见异常。根据中 枢性性早熟诊疗共识 ${ }^{[16]}$,女孩中枢性性早熟中 $80 \%$ 90\%为特发性,而男孩中枢性性早熟中 $66.7 \%$ 为继发性, 因此可进一步推测 Ras/MAPK通 
路相关疾病患儿出现中枢性性早熟或青春期发育 提前可能继发于该通路异常导致的 GnRH提前分 泌, 性早熟与PTPN11之间可能存在某种关联, 但 具体机制尚待进一步研究。

身材矮小是努南综合征主要临床特征之一， 亦是大部分努南综合征患儿就诊的原因。国外有 数据显示, 未接受治疗的努南综合征矮小患儿成 年终身高会低于 2 个标准差或第 3 百分位数 ${ }^{[3-5]}$ 。 因此, 对于努南综合征患儿身高矮小的治疗十分 重要。国外短期和长期研究数据显示, rhGH 治疗 可提高努南综合征矮小患儿的生长速率, 改善身 高 $^{[6-7]}$ 。MacFarlane 等 ${ }^{[17]}$ 开展了一项努南综合征 患儿rhGH治疗的对照研究, 该研究纳人 23 例治疗 和 8 例未治疗的努南综合征矮小患儿, 经过 3 年治 疗后, 治疗组比未治疗组平均身高增加 $3.3 \mathrm{~cm}$ 。 Horikawa 等 ${ }^{[18]}$ 通过一项多中心、随机、双盲、多剂 量 (低和高)平行对照试验评价 rhGH 治疗努南综 合征矮小患儿的疗效, 结果显示治疗 4 年后, 高剂 量组的身高获益明显。刘之慧等 ${ }^{[9]}$ 观察了 5 例努 南综合征患儿对 rhGH 治疗 1 年的反应, 发现患儿 在治疗期间生长速率明显增加。本文资料中, 7 例 努南综合征患儿 rhGH平均治疗时间为 (1.5 $0.8)$ 年, 治疗期间生长速率明显增加, 而 2 例规律 随访的未接受治疗的患儿仍保持低生长速率。

需要注意的是, 努南综合征患儿存在肿瘤及 肥厚型心肌病的潜在风险, 鉴于rhGH具有促有丝 分裂作用, 治疗期间须密切监测心脏功能及肿瘤 风险 ${ }^{[19]}$ 。已有研究中未见rhGH治疗努南综合征 出现严重不良反应的报道 ${ }^{[20]}$ 。本文资料中, 7 例 努南综合征患儿接受 $\mathrm{rhGH}$ 治疗后血清 IGF-1 水平 虽较治疗前上升,但仍处于正常范围。

综上所述,努南综合征临床表型谱广, 涉及全 身多个系统, 临床医生需提高警惕, 及时行基因检测 明确诊断。对于身高低于 2 个标准差或第 3 百分位 的努南综合征患儿, rhGH治疗可提高患儿生长速 率, 改善患儿身高,且治疗期间未见明显不良反应。

\section{利益冲突 所有作者均声明不存在利益冲突}

\section{参考文献}

[1] ROMANO A A, ALLANSON J E, DAHLGREN J, et al. Noonan syndrome: clinical features, diagnosis, and management guidelines $[\mathrm{J}]$. Pediatrics, 2010, 126(4): 746-759.
[2] ROMANO A A, DANA K, BAKKER B, et al. Growth response, near-adult height, and patterns of growth and puberty in patients with Noonan syndrome treated with growth hormone[J]. J Clin Endocrinol Metab, 2009, 94(7): 2338-2344.

[3] SHAW A C, KALIDAS K, CROSBY A H, et al. The natural history of Noonan syndrome: a long-term followup study $[\mathrm{J}]$. Arch Dis Childhood, 2006, 92(2): 128-132.

[4] NOONAN J A, RAAIJMAKERS R, HALL B D. Adult height in Noonan syndrome $[\mathrm{J}]$. Am J Med Genet, 2003, 123A(1): 68-71.

[5] YART A, EDOUARD T. Noonan syndrome : an update on growth and development $[\mathrm{J}]$. Curr Opin Endocrinol Diabetes Obes, 2018, 25(1): 67-73.

[6] AHMED M L, FOOT A B M, EDGE J A, et al. Noonan's syndrome: abnormalities of the growth hormone/IGF-I axis and the response to treatment with human biosynthetic growth Hormone $[\mathrm{J}]$. Acta Paediatrica, 1991, 80(4): 446-450.

[7] ŞIKLAR Z, GENENS M, POYRAZOLU Ş, et al. The growth characteristics of patients with Noonan syndrome: results of three years of growth hormone treatment: a nationwide multicenter study $[\mathrm{J}]$. J Clin Res Pediatr Endocrinol, 2016, 8(3): 305-312.

８］梁 雁. 基因重组人生长激素儿科临床规范应用的 建议 $[J]$. 中华儿科杂志, 2013, 51: 426-432.

LIANG Yan. Suggestions on the clinical application of genetic recombinant human growth hormone in pediatrics $[\mathrm{J}]$. Chinese Journal of Pediatrics, 2013, 51: 426-432. (in Chinese)

［9］刘之慧, 王林杰, 朱惠娟, 等. 6例Noonan 综合征患者 临床特点及重组人生长激素治疗效果分析 $[\mathrm{J}]$. 基础 医学与临床, 2017, 37 (2): 238-242.

LIU Zhihui, WANG Linjie, ZHU Huijuan, et al. Clinical characteristics and recombine growth hormone treatment efficacy in six patients with Noonan syndrome [J]. Basic and Clinical medicine, 2017, 37 (2): 238-242. (in Chinese)

[10 ] RICHARDS S, AZIZ N, BALE S, et al. Standards and guidelines for the interpretation of sequence variants: a joint consensus recommendation of the American College of Medical Genetics and Genomics and the Association for Molecular Pathology $[\mathrm{J}]$. Genet Med, 2015, 17(5): 405-423.

[11] RODRÍGUEZ F A, UNANUE N, HERNÁNDEZ M I, et al. Molecular characterization of Chilean patients with a clinical diagnosis of Noonan syndrome $[\mathbf{J}]$. J Pediatr Endocrinol Metab, 2014, 27(3-4): 305-309.

[12] ATIK T, AYKUT A, HAZAN F, et al. Mutation spectrum and phenotypic features in Noonan syndrome with PTPN11 mutations: definition of two novel mutations $[J]$. Indian J Pediatr, 2016, 83(6): 
517-521.

[13] CRONIN A S, HORAN T L, SPERGEL D J, et al. Neurotrophic effects of BDNF on embryonic gonadotropin-releasing hormone (GnRH) neurons $[\mathbf{J}]$. Eur J Neurosci, 2004, 20(2): 338-344.

[14] KERR B, DELRUE M A, SIGAUDY S, et al. Genotype-phenotype correlation in Costello syndrome: HRAS mutation analysis in 43 cases $[J]$. J Med Genet, 2006, 43(5): 401-405.

[15] VAN DER KAAY D C M, LEVINE B S, DOYLE D, et al. RASopathies are associated with delayed puberty; are they associated with precocious puberty too? $[\mathrm{J} / \mathrm{OL}]$. Pediatrics, 2016, 138(6): e20160182.

[16］中华医学会儿科学分会内分泌遗传代谢学组, 《中 华儿科杂志》编辑委员会. 中枢性性早熟诊断与治疗 共识(2015) [J]. 中华儿科杂志, 2015, 53(6): 412-418. Endocrinology, Genetics and Metabolism Group, Society of Pediatrics, Chinese Medical Association, Editorial Board of Chinese Journal of Pediatrics. Consensus on the diagnosis and treatment of central precocious puberty $(2015)[\mathrm{J}]$. Chinese Journal of
Pediatrics, 2015, 53(6): 412-418. (in Chinese)

[17] MACFARLANE C E, BROWN D C, JOHNSTON L B, et al. Growth hormone therapy and growth in children with noonan's syndrome: results of 3 years' follow-up $[\mathbf{J}]$. J Clin Endocrinol Metab, 2001, 86(5): 1953-1956.

[18] HORIKAWA R, OGATA T, MATSUBARA Y, et al. Long-term efficacy and safety of two doses of Norditropin $^{\circledR}$ (somatropin) in Noonan syndrome: a 4year randomized, double-blind, multicenter trial in Japanese patients $[J]$. Endocr J, 2020, 67(8): 803-818.

[19] RANKE M B, HEIDEMANN P, KNUPFER C, et al. Noonan syndrome: growth and clinical manifestations in 144 cases $[\mathrm{J}]$. Eur J Pediatr, 1988, 148(3): 220-227.

[20 ] RODRÍGUEZ F, GAETE X, CASSORLA F. Etiology and treatment of growth delay in Noonan syndrome $[\mathrm{J}]$. Front Endocrinol, 2021, 12: 691240.

[本文编辑 沈 敏 余 方]

\section{刘冲研究员团队研究成果揭开少突胶质前体细胞发育起源 与异质性的关系}

2021年8月16日，《先进科学》(Advanced Science)在线发表了浙江大学医学院刘冲研究员团队关于少突胶质前体细 胞 (OPC) 的发育起源与异质性的关系的最新研究成果 “In vivo clonal analysis reveals development heterogeneity of oligodendrocyte precursor cells derived from distinct germinal zones” (https://doi. org/10.1002/advs. 202102274)。该研究 建立了针对OPC谱系的体内克隆分析技术, 并利用该技术发现背侧和腹侧两种干细胞起源的OPC命运截然不同,证明了 发育起源对于OPC命运具有决定性作用。

研究人员结合基因工程修饰小鼠、胚胎电转技术和PiggyBac转座子技术发展了一套全新的细胞特异性的体内克隆 分析系统, 可以实现在特定发育时间窗口对特定细胞类型进行谱系示踪与单细胞起源的克隆分析。通过改变胚胎电转 的电流方向, 把转座子上的DNA序列稳定整合到胚胎期小鼠侧脑室不同区域的神经干细胞中,结合在OPC中特异表达 Cre重组酶的NG2-Cre/CreER转基因小鼠,实现对不同起源OPC及其子代细胞的标记和追踪。研究人员分别在群体水平 和克隆水平探究背侧和腹侧起源的OPC分化、增殖、分布等细胞行为,发现背侧起源的OPC在成年后持续扩增并保持较高 的分化比例, 而腹侧起源的OPC虽然在早期与背侧起源的OPC有相似的细胞行为,但成年后逐渐调亡,至小鼠 6 个月时基 本全部消失, 且相较于背侧起源的OPC保持更低的分化比例。研究人员还通过数学建模分析了 OPC早期克隆的大小,推 断背侧和腹侧起源的OPC的不同分裂模式。研究人员通过Crispr-Cas9技术在OPC克隆中特异性敲除了 $N F 1$ 基因,逆转了 腹侧起源的OPC成年后逐渐凋亡的命运。综上所述,该研究通过对不同起源OPC的克隆分析,揭示了 OPC发育起源对细 胞异质性和细胞命运的决定性作用,并找出了改变腹侧起源OPC凋亡命运的关键基因,为通过OPC移植治疗脱髓鞘疾病 提供了新的思路。

刘睿博士研究生为论文第一作者。研究获得国家自然科学基金、国家重点研发计划等资助。 